Notfall Rettungsmed 2011 · 14:441-442

DOI 10.1007/s10049-011-1426-z

Online publiziert: 24. September 2011

(c) Springer-Verlag 2011

\author{
U. Kreimeier ${ }^{1}$. C. Waydhas ${ }^{2}$ \\ ${ }^{1}$ Klinik für Anaesthesiologie, Klinikum der Universität \\ München, Campus Innenstadt, München \\ ${ }^{2}$ Klinik und Poliklinik für Unfallchirurgie, Universitätsklinikum Essen
}

\title{
Medizinjuristische Aspekte der Notfallmedizin
}

Medikolegale Erwägungen halten im täglichen ärztlichen Handeln weiterhin zunehmend Einzug. Sie sind meist nicht so sehr im konkreten Einzelfall von vordergründiger Bedeutung, sondern bestimmen vor allem grundsätzliches Handeln und organisatorische Vorkehrungen. Dies trifft auch für den Rettungsdienst und die Notfallversorgung zu, auch wenn die Anzahl juristisch ausgetragener Konflikte in diesem Sektor vergleichsweise gering ist.

Erschwert wird die Situation für den Notarzt, den Rettungsassistenten oder das weitere medizinische Personal durch zahlreiche Schnittstellen zwischen den unterschiedlichsten beteiligten Personenkreisen. Hinzu kommt der Umstand, dass meist unter hohem Zeitdruck kritische, für die Patienten lebensbestimmende Entscheidungen zu treffen sind, die sich im juristischen, ethischen und auch medizinisch-fachlichen Grenzbereich befinden. Die Mischung aus unvollständigem und bei verschiedenen Berufsgruppen unterschiedlichem Wissen über gesetzliche Regelungen und deren Auslegung und Rahmenbedingungen, verbunden mit objektiven Defiziten in den Gesetzen und Bestimmungen, führen nicht selten zu Verunsicherungen beim ärztlichen und nichtärztlichen Rettungsdienstpersonal.

\section{Unvollständiges Wissen über gesetzliche Regelungen führt zu Verunsicherungen beim Rettungsdienstpersonal}

Mit den 4 Beiträgen zum vorliegenden Leitthema „Medizinjuristische Aspekte der Notfallmedizin“ soll versucht werden, in einigen ausgewählten Aspekten den aktuellen Wissens- und Gesetzesstand unter Berücksichtigung der Umsetzung in der konkreten Situation darzustellen und Handlungshilfen zu geben. Zusätzlich werden aber auch Bereiche benannt, in denen es nicht möglich ist, dezidierte Handlungsschritte oder Entscheidungen vorzugeben. Anforderungen an die Gesetzgebung oder Lösungswege für ungeklärte Fragen werden modellhaft angeschnitten, ohne dass ein Anspruch auf Vollständigkeit bestehen könnte.

Durch die Einladung ärztlicher Autoren soll ein möglichst hoher Grad an Relevanz für den rettungsdienstlichen Alltag, durch die Einbindung rechtsanwaltlicher Gutachter die juristische Korrektheit erreicht werden. Den juristischen Kollegen im Herausgeberboard der Zeitschrift sei an dieser Stelle ausdrücklich dafür gedankt.

Der Beitrag von Hummes, Hartmann und Pajonk setzt sich mit der Feststellung der Einwilligungsfähigkeit bzw. der Unfähigkeit zur Einwilligung und anderer Rahmenbedingungen auseinander, die auch Voraussetzung für die Anwendung von Zwangsmaßnahmen sind. In dem Artikel wird auf den Unterschied zwischen Geschäftsfähigkeit und Einwilligungsfähigkeit eingegangen und dargelegt, anhand welcher Kriterien letztere fest gemacht werden kann. So wird beschrieben, welche Anhaltspunkte ein Maß an Selbst- oder Fremdgefährdung anzeigen, das eine Behandlung oder Unterbringung gegen den Willen des Patienten und ohne richterliche Entscheidung erlaubt oder sogar erforderlich macht.

Einem gänzlich anderen Bereich widmen sich die Beiträge von Frau Kollegin Kloke und der Autorengruppe in der Schmitten et al. Sie beschäftigen sich mit den Lücken im neuen Patientenverfügungsgesetz von 2009 und wie diese geschlossen werden könnten. Sehr schnell hat sich nach der Veröffentlichung gezeigt, dass das Gesetz nicht für jede erdenkliche Notfallsituation, in der eine sofortige, für den Patienten weit reichende Entscheidung zu treffen ist, konkrete Regelungen vorgeben kann. Erschwert wird die Entscheidung über die definitiv $\mathrm{zu}$ wählende medizinische Vorgehensweise vor Ort, wenn man sich die Situation vor Augen führt, in der Notarzt und Rettungsdienstpersonal vom juristisch relevanten Inhalt einer Patientenverfügung Kenntnis erhalten: Unter dem gegebenen emotionalen und zeitlichen Druck sollen sie ad hoc medizinisch korrekt und nach den von Fachgesellschaften und Berufsgruppen konsentierten Leitlinien oder Empfehlungen handeln. Im Rettungsdienst wird dies fast immer durch den Umstand belastet, dass die Behandelnden erstmals mit den Patienten und mit deren Umfeld konfrontiert werden.

In den Beiträgen werden die Regelungen dargestellt, die für (Not-)Arzt und nichtärztliches Rettungsdienstpersonal verbindlich sind, ebenso wird aufgezeigt, wo klare und anwendbare Vorgaben nicht existieren. Für solche Situationen kann und darf der Leser keine verbindliche Empfehlung erwarten. Aller- 
dings werden Lösungsansätze aufgezeigt, die in Planung oder bereits in konkreter Umsetzung sind. Sie können Anregung geben, im eigenen Tätigkeitsbereich ähnliche Projekte anzustoßen.

Ihre

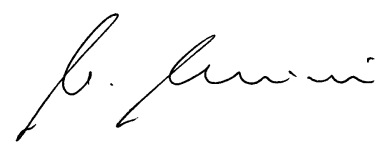

U. Kreimeier

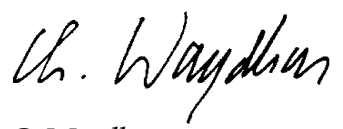

C. Waydhas

\section{Korrespondenzadressen}

\section{Prof. Dr. U. Kreimeier}

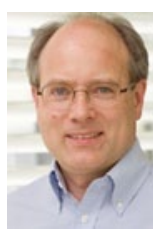

Klinik für Anaesthesiologie,

Klinikum der Universität

München, Campus Innenstadt

Nussbaumstraße 20,

80336 München

Uwe.Kreimeier@med.

uni-muenchen.de

\section{Prof. Dr. C. Waydhas}

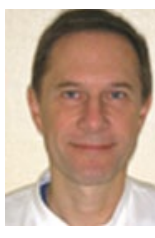

Klinik und Poliklinik

für Unfallchirurgie,

Universitätsklinikum Essen

Hufelandstraße 55,

45122 Essen

christian.waydhas@

uni-essen.de
„Notfall + Rettungsmedizin“ bietet Ihnen 8-mal im Jahr umfassende und aktuelle Beiträge zu interessanten Themenschwerpunkten (Leitthemen) aus allen Bereichen der Notfallmedizin.

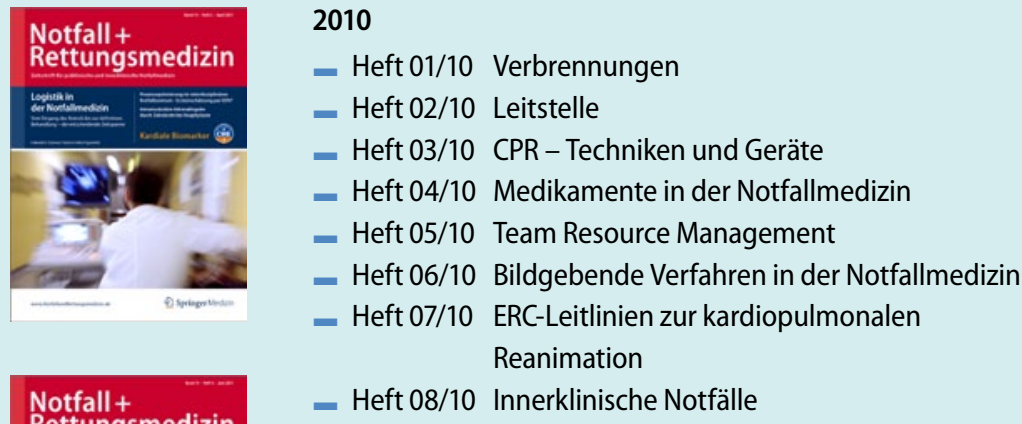

2011

- Heft 01/11 Präklinisches Atemwegsmanagement

- Heft 02/11 Lebensgefährliche Herzrhythmusstörungen

- Heft 03/11 Notfallmedizin und Informationstechnologie

- Heft 04/11 Leichte Schädel-Hirn-Traumata

- Heft 05/11 Notaufnahme

- Heft 06/11 Medizinrechtliche Aspekte der Notfallmedizin

Notfall +

Rettungsmedizin

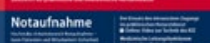

- Heft 07/11 Pädiatrische Notfälle

- Heft 08/11 Advanced Technology in der Notfallmedizin

\section{Alle Beiträge auf einen Blick}

Abonnenten haben online Zugriff auf alle Beiträge im elektronischen Volltextarchiv unter

\section{www.NotfallundRettungsmedizin.de}

Ins Volltextarchiv gelangen Sie von der Startseite über den Navigationspunkt "Online-Archiv".

Eine interessante Lektüre wünscht lhnen

Ihre Redaktion „Notfall + Rettungsmedizin" 